\title{
Speckle POF sensor for Detecting Vital Signs of Patients
}

\author{
M. Lomer*, L. Rodriguez-Cobo, P. Revilla, G. Herrero, F. Madruga, J.M. Lopez-Higuera \\ Photonics Engineering Group, University of Cantabria, 39005, Santander (Spain)
}

\begin{abstract}
In this work, both arterial pulse and respiratory rate have been successfully measured based on changes in speckle patterns of multimode fibers. Using two fiber-based transducers, one located on the wrist and another in the chest, both disturbances were transmitted to the fiber, varying the speckle pattern. These variations of the speckle pattern were captured using a commercial webcam and further processed using different methods. The achieved results have been presented and the simultaneous monitoring of both vital signs has been also discussed. The feasibility to use the proposed sensor system for this application is demonstrated.
\end{abstract}

Keywords: Speckle patterns, optical fiber sensor, arterial pulse and respiration rate sensing.

\section{INTRODUCTION}

The application of optical fibers sensors for medicine has been increased during recent years. Indeed, many properties of optical fibers such as, flexibility and adaptability, small dimension and low weight and immunity to electromagnetic interferences make them very attractive to measure biomedical parameters [1]. Different optical fiber sensing technologies have been successfully applied to measure vital signs of human beings. Examples such as monitoring the heart rate, the breathing process or even the physical movement of the body have been reported based on intensity and phase modulation or spectral shifts [2-7].

Particularly, an intensity-based transducer to detect the changes on the thorax diameter to measure the respiratory rate has been reported [2]. This solution, based on placing a headband around the chest, was simple, but it was too large for a continuum monitoring process. Other approach, based on phase modulation, employed a transducer placed near to the patient and other that records lung and heart induced movements to be combined into an interferometric setup, generating a signal varying over time [3-4]. The main drawback of this scheme was the signal processing method, which had to be capable of isolating both respiration and cardiac activities. Spectral displacement sensors (e.g. FBGs) have been also tested for these purposed, obtaining simultaneous measurements of heart and respiratory rates [5-6], but they associated cost are high and usually employ glass-fibers. All the described methods require optical arrays and other sophisticated components, besides specific signal processing schemes to obtain both respiration and heart rates. A heart rate sensor based on measuring the arterial pulse with Fiber Specklegram sensors have been previously demonstrated [7], showing the benefits of this technology for its application in medical scenarios.

In this work, a very low cost sensor to obtain the simultaneous measurement of heart and respiratory rates with polymer optical fibers (POF) using speckle patterns has been proposed. Based on $240 \mu \mathrm{m}$ diameter core POFs, the speckle patterns has been recorded using a commercial webcam to obtain simultaneous signals of different sensing points, allowing a better analysis of vital parameters.

\section{SENSOR CONCEPT}

Speckle patterns can be obtained at multimode fibers output when coherent light propagates inside. This interferometric process is highly sensitive to environmental perturbations, where each speckle fluctuates but the intensity distribution of the speckle pattern observed on the screen should remain constant. An approximation to the speckle phenomenon in optical fibers is attributed to random inter-modal and self-modal interactions [8]. The amount of speckle is proportional to the number of modes propagated by the fiber and each mode has a different phase velocity. When a perturbation is applied to the fiber (e.g. acoustic wave), the speckle pattern changes. These changes can be recorded by a video camera and then, with a proper processing method, a representative signal correlates to the perturbation can be obtained. In this work, this approach has been employed to measure the arterial pulse and respiration rate.

*lomerm@unican.es

23rd International Conference on Optical Fibre Sensors, edited by José Miguel López-Higuera, Julian Jones, Manuel López-Amo, José Luis Santos, Proc. of SPIE Vol. 9157, 91572

(C) 2014 SPIE · CCC code: 0277-786X/14/\$18 · doi: 10.1117/12.2059747 
In the first case, the heart pumps blood that flows through the arteries and causes a pulse that propagates as an acoustic wave. By a simple palpation of the skin close to the arteries (e.g. wrist or neck), the heart pump sequence can be detected. On the contrary, the breathing process (inhalation, exhalation) produces changes in the chest diameter. By placing a multimode optical fiber in each of these locations, both mechanical variations affect the interference process within the optical fiber, modifying the speckle pattern at the fiber output.

The pixels of a CCD camera are individual, being able to register multiple small images projected to the same surface and process them simultaneously. A typical webcam has a CCD area of $1 \times 1 \mathrm{~mm}^{2}$ and if the projected spot of a $240 \mu \mathrm{m}$ fiber is around $50 \mu \mathrm{m}^{2}$, several fibers can be projected on the same CCD area recording individual signals of different sensing points. In Fig. 1, the sensor system is illustrated: different points of the human body can be simultaneously measured using the changes in the speckle patterns.

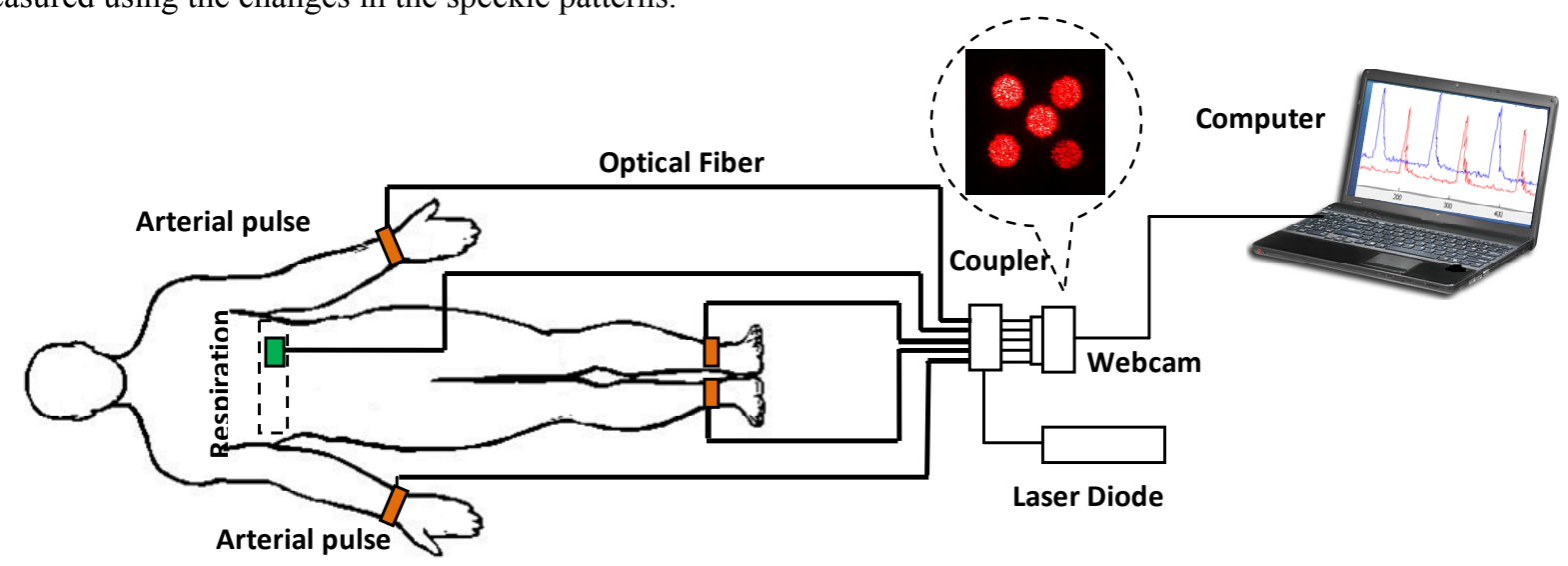

Fig. 1. Illustration of the proposed system to the measurement of arterial pulse and respiration rate.

\section{EXPERIMENTS AND RESULTS}

It has been proposed to two different types of transducers, one to measure the arterial pulse and other to measure the respiration rate. Both transducers have been manufactured using POF of $250 \mu \mathrm{m}$ of external diameter (core diameter of $240 \mu \mathrm{m}$ ). The materials of the core and the cladding were polymethyl-methacrylate (PMMA) and fluorinated polymer, respectively. The refractive indices of the core and the cladding were 1.49 and 1.40 , respectively, and the numerical aperture was around 0.5. A cheap laser diode $(\lambda=620 \mathrm{~nm})$ with a coherence length of $200 \mu \mathrm{m}$ has been employed as light source.

In the transducer to measure arterial pulse, the fiber was attached to a headband and placed around a wrist, maintaining a free region of the fiber in direct contact to the skin near the artery. The mechanical waves generated by the heart pump, locally disturbs the fiber, modifying the propagated modes and, consequently, varying the speckle pattern at the fiber exit. The second transducer (to measure the respiration) was based on attaching a POF to an elastic cord, which was located around the chest. The central region of the fiber had an U-shaped bend with a radius of $5 \mathrm{~mm}$. The changes in the chest diameter due to respiration modified the bend radius of the central region, altering the speckle pattern on the fiber output.

The experimental measurements have been performed over 20 people aged between 20 and 28 years, comprising both women and men. The arterial pulse has been measured by placing a wrist over a portion of fiber located on a rigid support. The respiration rate has been measured with a piece of fiber embedded into the elastic band located around the chest. The images of speckle patterns have been recorded using a webcam and processed in real time using Matlab.

\subsection{Experimental results: arterial pulse and respiration rate.}

The results of the measurements of the arterial pulse are plotted as the difference between frames and function of time. Each person has been measured for 30 seconds and repeated 3 times and pulse are measured as beats per minute (BPM). 
The achieved results are comprised between 61 and 78 BPM. 10 seconds of the sequences of these two extremes are shown in Figures $2(a, b)$. Arterial pulse profiles are of good quality and very regular. The waveforms of the pulses of the hand have similar profiles but with variations in the amplitude. The proposed scheme has been compared to a traditional method (by palpation), exhibiting an averaged error of \pm 0.15 BPM.

Similar procedures have been followed to measure the respiration rate. According to the procedure described in Section 3 , respiration measures have been performed on each person for 60 seconds and repeated 3 times. The mean respiratory rate has been measured in breaths per minute. The results obtained of respiration rate were within the range of the adult population. In Figure 3(a,b), 30 seconds of different signals of the breathing process of two people have been depicted, obtaining 12 and 17 breaths per minute (bpm), respectively. The waveform of respiration rates exhibited good inhalationexhalation cycles.

\subsection{Simultaneous measurement of multiple points.}

Trying to obtain simultaneous measurements of different parameters using the same CCD, two speckle patterns from two separated fibers have been recorded by a single webcam. In Figure 4(a), a capture of two speckle patterns captured using the same webcam are depicted. Three regions to be analyzed have been defined within the images. In those regions (labeled with A, B and C) the speckle patterns have been analyzed by calculating the difference between frames. The achieved results for different perturbations applied to both fibers have been depicted in Figure 4 (b). It can be noticed that the two speckle patterns (regions A and C) do not interfere, keeping their own perturbations. The dark region (region B) is not illuminated by the speckle pattern, thus it remains constant without fluctuations. This scheme can be also employed for medical purposes, being able to obtain both heart and respiration rates simultaneously when combining POFs of smaller diameters.
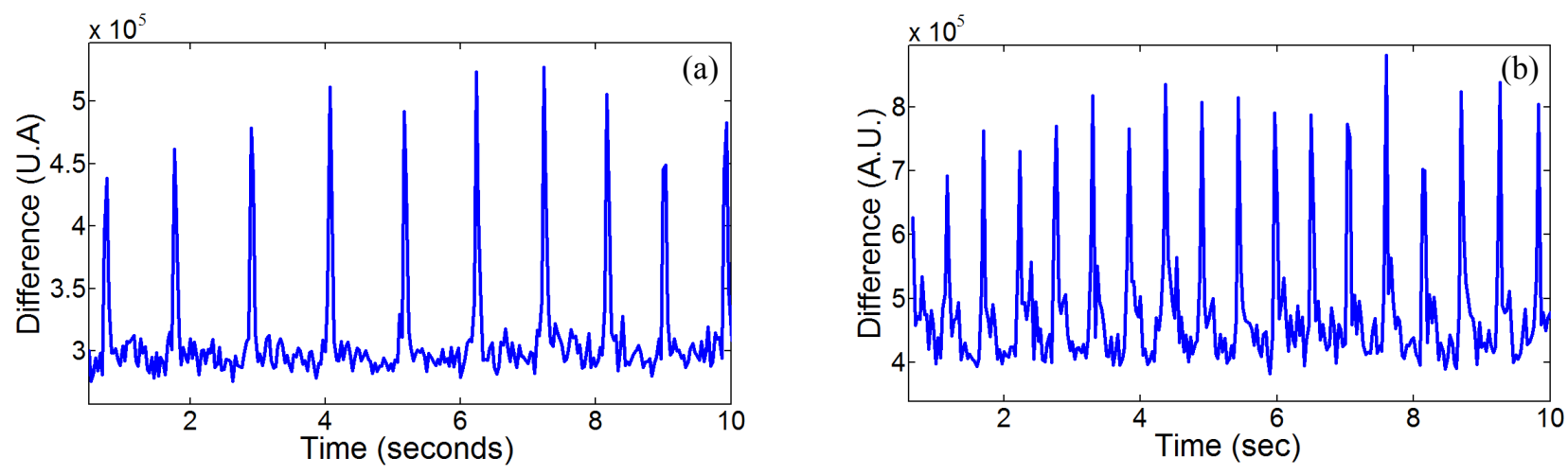

Figure 2. Results of measurement of blood pulse into two people using speckle fiber, a) 61 BPM, and b) 78 BPM.
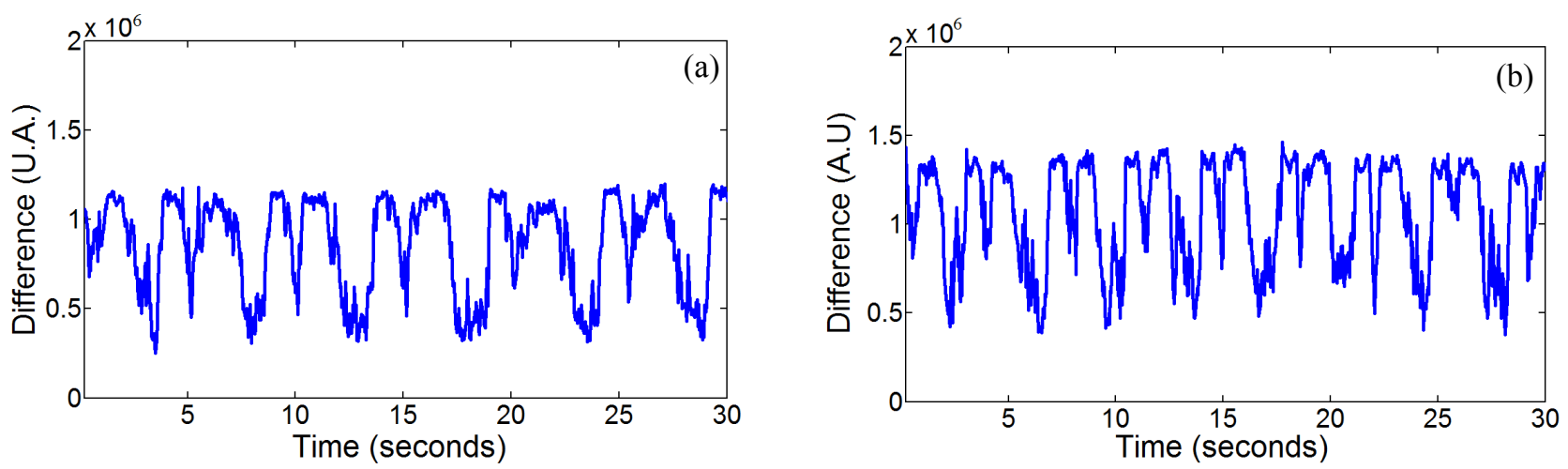

Figure 3. As a result of the respiratory rate using speckle fiber, a) $12 \mathrm{bpm}$, b) $17 \mathrm{bpm}$. 


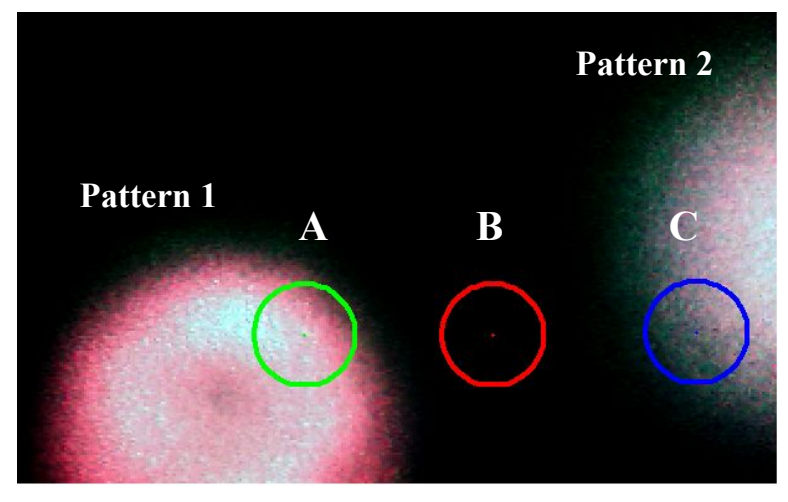

(a)

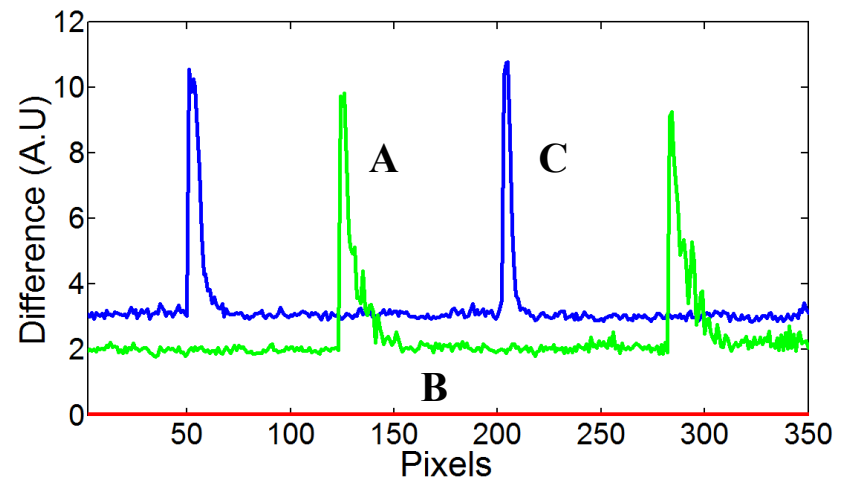

(b)

Figure 3. Simultaneous measurement of two speckle patterns recorded by a webcam.

\section{CONCLUSIONS}

In this work, both arterial pulse and respiratory rates have been experimentally measured using fiber specklegram sensors. Only employing a cheap webcam capturing at 30 frames per second as a detecting device, both waveforms proportional to each measurand have been obtained. Combining the high sensitivity of fiber specklegram sensors and a suitable design could provide more information on the detected vital signs. Besides, the possibility of capturing different speckle patterns placed on different regions of a single camera has been also demonstrated. These results allow the increase in the number of sensing points, achieving low cost systems able to obtain different parameters measured at different points.

This work has been supported by the project TEC2010-20224-C02-02 and grant AP2009-1403. The authors thank Victor Landeras by measurements of respiratory rate.

\section{REFERENCES}

[1] A.G. Mignani and F. Baldini, "In-vivo biomedical monitoring by fiber-optic systems", J. Lightwave Technol., 13, 1396-1406 (1995).

[2] A. Babchenko, B. Khanokh, Y. Shomer, and M. Nitzan, "Fiber optic sensor for the measurement of respiratory chest circumference changes", J. Biomed. Opt., 4(2), 224-229 (1999).

[3] S. Šprager and D. Zazula, "Heartbeat and respiration detection from optical interferometric signals by using a multimethod approach", IEEE Trans. Biomed. Eng. 59(10), 2922-2929 (2012).

[4] F. C. Favero, J. Villatoro, and V. Pruneri, "Microstructured optical fiber interferometric breathing sensor," J. Biomed. Opt. 17(3), 037006 (2012).

[5] A.F. Silva, J.P. Carmo, P.M. Mendes and J.H. Correia, "Simultaneous cardiac and respiratory frequency measurement based on a single fiber Bragg grating sensor", Meas. Sci. Technol. 22, 075801 (2011).

[6] L. Dziuda et al., "Fiber Bragg grating-based sensor for monitoring respiration and heart activity during magnetic resonance imaging examinations", J. Biomed. Opt. 18(5), 057006 (2013).

[7] M. Lomer, L. Rodriguez-Cobo, J.M. Lopez-Higuera, "Plastic optical fiber sensor for measuring arterial pulse", Proceeding, The 21th Int. Conference on Plastic Optical Fibers, September 10-12, Atlanta, USA (2012).

[8] W.B. Spillman,.Jr., B.R. Kline, L.B. Maurice, and P.L. Fuhr, "Statistical-mode sensor for fiber optic vibration sensing uses", Applied Optics, 28, 3166-3176, (1989). 